\title{
MULTIPLE SETS EXPONENTIAL CONCENTRATION AND HIGHER ORDER EIGENVALUES
}

\author{
NATHAËL GOZLAN \& RONAN HERRY
}

\begin{abstract}
On a generic metric measured space, we introduce a notion of improved concentration of measure that takes into account the parallel enlargement of $k$ distinct sets. We show that the $k$-th eigenvalues of the metric Laplacian gives exponential improved concentration with $k$ sets. On compact Riemannian manifolds, this allows us to recover estimates on the eigenvalues of the Laplace-Beltrami operator in the spirit of an inequality of [11].
\end{abstract}

\section{Contents}

Introduction

1. Multiple sets exponential concentration in abstract spaces

1.1. Courant-Fischer formula and generalized eigenvalues in metric spaces

1.2. Statement of the main results

1.3. Proofs

1.4. Two more multi-set concentration bounds

1.5. Comparison with the result of Chung-Grigor'yan-Yau 10

2. Eigenvalue estimates for non-negatively curved spaces 11

3. Extension to Markov chains 12

4. Functional forms of the multiple sets concentration property 13

5. Open questions $\quad 15$

5.1. Gaussian multi-set concentration 15

5.2. Equivalence between multi-set concentration and lower bounds on $\begin{array}{ll}\text { eigenvalues in non-negative curvature } & 15\end{array}$

References

\section{INTRODUCTION}

Let $(M, g)$ be a smooth compact connected Riemannian manifold with its normalized volume measure $\mu$ and its geodesic distance $d$. The Laplace-Beltrami operator $\Delta$ is then a non-positive operator whose spectrum is discrete. Let us denote by $\lambda^{(k)}, k=$ $0,1,2 \ldots$, the eigenvalues of $-\Delta$ written in increasing order. With these notations $\lambda^{(0)}=0$ (achieved for constant functions) and (by connectedness) $\lambda^{(1)}>0$ is the socalled spectral gap of $M$.

The study of the spectral gap of Riemannian manifolds is, by now, a very classical topic which has found important connections with numerous geometrical and analytical questions and properties. The spectral gap constant $\lambda^{(1)}$ is for instance related to Poincaré type inequalities and governs the speed of convergence of the heat flow to equilibrium. It is also related to Ricci curvature via the classical Lichnerowicz theorem [20] and to Cheeger isoperimetric constant via Buser's theorem [7]. We refer to [5, 8] and the references therein for a complete picture. 
Another important property of the spectral gap constant, first observed by Gromov and Milman [16], is that it controls exponential concentration of measure phenomenon for the reference measure $\mu$. The result states as follows. Define for all Borel sets $A \subset M$, its $r$-enlargement $A_{r}$ as the (open) set of all $x \in E$ such that there exists $y \in A$ with $d(x, y)<r$. Then, for any $A \subset M$ such that $\mu(A) \geq 1 / 2$ it holds

$$
\mu\left(A_{r}\right) \geq 1-b e^{-a \sqrt{\lambda^{(1)}} r}, \quad \forall r>0,
$$

where $a, b>0$ are some universal constants (according to [19, Theorem 3.1], one can take $b=1$ and $a=1 / 3)$. Note that this implication is very general and holds on any metric space supporting a Poincaré inequality (see [19, Corollary 3.2]). See also [6, 26, 1, 15] for alternative derivations, generalizations or refinements of this result.

This note is devoted to a multiple sets extension of the above result. Roughly speaking, we will see that if $A_{1}, \ldots, A_{k}$ are sets which are pairwise separated in the sense that $d\left(A_{i}, A_{j}\right):=\inf \left\{d(x, y): x \in A_{i}, y \in A_{j}\right\}>0$ for any $i \neq j$ and $A$ is their union then the probability of $A_{r}$ goes exponentially fast to 1 at a rate given by $\sqrt{\lambda^{(k)}}$ as soon as $r$ is such that the sets $A_{i, r}, i=1, \ldots, k$ remain separated. More precisely, it follows from Theorem 1.1 (whose setting is actually more general) that, if $A_{1}, \ldots, A_{k}$ are such that $\mu\left(A_{i}\right) \geq \frac{1}{k+1}$ and $d\left(A_{i, r}, A_{j, r}\right)>0$ for all $i \neq j$, then, denoting $A=A_{1} \cup \ldots \cup A_{k}$, it holds

$$
\mu\left(A_{r}\right) \geq 1-\frac{1}{k+1} \exp \left(-c \min \left(r^{2} \lambda^{(k)} ; r \sqrt{\lambda^{(k)}}\right)\right),
$$

for some universal constant $c$. This kind of probability estimates first appeared, in a slightly different but essentially equivalent formulation in the work of Chung, Grigor'yan and Yau [11, 10] (see also the related paper [12] by Friedman and Tillich). Nevertheless, the method of proof we use to arrive at (0.1) (based on the Courant-Fischer min-max formula for the $\lambda^{(k)}$ 's) is quite different from the one of [11, 10] and seems more elementary and general. This is discussed in details in Section 1.5.

The paper is organized as follows. In Section 1, we prove (0.1) in an abstract metric space framework. This framework contains, in particular, the compact Riemannian case equipped with the Laplace operator presented above. The Section 1.5 contains a detailed discussion of our result with the one of Chung, Grigor'yan \& Yau. In Section 2, we recall various bounds on eigenvalues on several non-negatively curved manifolds. Section 3 gives an extension of (0.1) to discrete Markov chains on graphs. In Section 4, we give a functional formulation of the results of Sections 1 and 3. As a corollary of this functional formulation, we obtain a deviation inequality as well as an estimate for difference of two Lipschitz extensions of a Lipschitz function given on $k$ subsets. Finally, Section 5 discusses open questions related to this type of concentration of measure phenomenon.

\section{Multiple sets exponential CONCEntration in ABStract SPaCES}

1.1. Courant-Fischer formula and generalized eigenvalues in metric spaces. Let us recall the classical Courant-Fischer min-max formula for the $k$-th eigenvalue $(k \in \mathbb{N})$ of $-\Delta$, noted $\lambda^{(k)}$, on a compact Riemannian manifold $(M, g)$ equipped with its (normalized) volume measure $\mu$ :

$$
\lambda^{(k)}=\inf _{\substack{V \subset \mathcal{C}^{\infty}(M) \\ \operatorname{dim} V=k+1}} \sup _{f \in V \backslash\{0\}} \frac{\int|\nabla f|^{2} \mathrm{~d} \mu}{\int f^{2} \mathrm{~d} \mu},
$$

where $\nabla f$ is the Riemannian gradient, defined through the Riemannian metric $g$ (see e.g [8]) and $|\nabla f|^{2}=g(\nabla f, \nabla f)$. The formula (1.1) above does not make explicitly reference to the differential operator $\Delta$. It can be therefore easily generalized to a more abstract setting, as we shall see below. 
In all what follows, $(E, d)$ is a complete, separable metric space and $\mu$ a reference Borel probability measure on $E$. Following [9], for any function $f: E \rightarrow \mathbb{R}$ and $x \in E$, we denote by $|\nabla f|(x)$ the local Lipschitz constant of $f$ at $x$, defined by

$$
|\nabla f|(x)=\left\{\begin{array}{l}
0 \text { if } x \text { is isolated } \\
\limsup _{y \rightarrow x} \frac{|f(x)-f(y)|}{d(x, y)} \text { otherwise. }
\end{array}\right.
$$

Note that when $E$ is a smooth Riemannian manifold, equipped with its geodesic distance $d$, then, the local Lipschitz constant of a differentiable function $f$ at $x$ coincides with the norm of $\nabla f(x)$ in the tangent space $T_{x} E$. With this notion in hand, a natural generalization of (1.1) is as follows (we follow [23, Definition 3.1]):

$$
\lambda_{d, \mu}^{(k)}:=\inf _{\substack{V \subset H^{1}(\mu) \\ \operatorname{dim} V=k+1}} \sup _{f \in V \backslash\{0\}} \frac{\int|\nabla f|^{2} \mathrm{~d} \mu}{\int f^{2} \mathrm{~d} \mu}, \quad k \geq 0,
$$

where $H^{1}(\mu)$ denotes the space of functions $f \in L^{2}(\mu)$ such that $\int|\nabla f|^{2} \mathrm{~d} \mu<+\infty$. In order to avoid heavy notations, we drop the subscript and we simply write $\lambda^{(k)}$ instead of $\lambda_{d, \mu}^{(k)}$ within this section.

1.2. Statement of the main results. To state our first main result, we need further notations: for any $k \geq 1$, we denote by $\Delta_{k}$ the set of vectors $\left(a_{1}, \ldots, a_{k}\right) \in[0,1]^{k}$ satisfying the following linear constraints

$$
\sum_{j=1}^{k} a_{j} \leq 1 \quad \text { and } \quad a_{i}+\sum_{j=1}^{k} a_{j} \geq 1, \forall i \in\{1, \ldots, k\} .
$$

Recall the classical notation $d(A, B)=\inf \{d(x, y): x \in A, y \in B\}$ of the distance between two sets $A, B \subset E$.

The following theorem is the main result of the paper and is proved in Section 1.3.

Theorem 1.1. There exists a universal constant $c>0$ such that, for any $k \geq 1$ and for all sets $A_{1}, \ldots, A_{k} \subset E$ such that $\min _{i \neq j} d\left(A_{i}, A_{j}\right)>0$ and $\left(\mu\left(A_{1}\right), \ldots, \mu\left(A_{k}\right)\right) \in \Delta_{k}$, the set $A=A_{1} \cup A_{2} \cup \cdots \cup A_{k}$ satisfies

$$
\mu\left(A_{r}\right) \geq 1-(1-\mu(A)) \exp \left(-c \min \left(r^{2} \lambda^{(k)} ; r \sqrt{\lambda^{(k)}}\right)\right),
$$

for all $0<r \leq \frac{1}{2} \min _{i \neq j} d\left(A_{i}, A_{j}\right)$, where $\lambda^{(k)} \geq 0$ is defined by (1.2).

Note that, since $(1 /(k+1), \ldots, 1 /(k+1)) \in \Delta_{k}$, Theorem 1.1 immediately implies Inequality (0.1).

Inverting our concentration estimate, we obtain the following statement that provides a bound on the $\lambda^{(k)}$ 's.

Proposition 1.2. Let $(E, d, \mu)$ be a metric measured space and $\lambda^{(k)}$ be defined as in (1.2). Let $A_{1}, \ldots, A_{k}$ be measurable sets such that $\left(\mu\left(A_{1}\right), \ldots, \mu\left(A_{k}\right)\right) \in \Delta_{k}$, then, with $r=\frac{1}{2} \min _{i \neq j} d\left(A_{i}, A_{j}\right)$ and $A_{0}=E \backslash\left(\cup A_{i}\right)_{r}$,

$$
\lambda^{(k)} \leq \frac{1}{r^{2}} \psi\left(\frac{1}{c} \min _{i} \ln \frac{\mu\left(A_{i}\right)}{\mu\left(A_{0}\right)}\right),
$$

where $\psi(x)=\max \left(x, x^{2}\right)$.

Proof. Let $A=\cup_{i} A_{i}$. Inverting the formula in Theorem 1.1, we obtain

$$
\lambda^{(k)} \leq \frac{1}{r^{2}} \psi\left(\frac{1}{c} \ln \frac{1-\mu(A)}{1-\mu\left(A_{r}\right)}\right),
$$


where $\psi(x)=\max \left(x, x^{2}\right)$. By definition of $\Delta_{k}$,

$$
1-\mu(A)=1-\sum_{i} \mu\left(A_{i}\right) \leq \min _{i} \mu\left(A_{i}\right) .
$$

Therefore, letting $A_{0}=E \backslash A_{r}$, we obtain the announced inequality by non-decreasing monotonicity of $\psi$ and $\ln$.

The collection of sets $\Delta_{k}, k \geq 1$ has the following useful stability property:

Lemma 1.3. Let $I_{1}, I_{2}, \ldots, I_{n}$ be a partition of $\{1, \ldots, k\}, k \geq 1$. Let $a=\left(a_{1}, \ldots, a_{k}\right) \in$ $\mathbb{R}^{k}$ and define $b=\left(b_{1}, \ldots, b_{n}\right) \in \mathbb{R}^{n}$ by setting $b_{i}=\sum_{j \in I_{i}} a_{j}, i \in\{1, \ldots, n\}$. If $a \in \Delta_{k}$ then $b \in \Delta_{n}$.

Proof. The proof is obvious and left to the reader.

Thanks to this lemma it is possible to iterate Theorem 1.1 and to obtain a general bound for $\mu\left(A_{r}\right)$ for all values of $r>0$. This bound will depend on the way the sets $A_{1, r}, \ldots, A_{k, r}$ coalesce as $r$ increases. This is made precise in the following definition.

Definition 1.1 (Coalescence graph of a family of sets). Let $A_{1}, \ldots, A_{k}$ be subsets of $E$. The coalescence graph of this family of sets is the family of graphs $G_{r}=\left(V, E_{r}\right)$, $r>0$, where $V=\{1,2, \ldots, k\}$ and the set of edges $E_{r}$ is defined as follows: $\{i, j\} \in E_{r}$ if $d\left(A_{i, r}, A_{j, r}\right)=0$.

Corollary 1.4. Let $A_{1}, \ldots, A_{k}$ be subsets of $E$ such that $\min _{i \neq j} d\left(A_{i}, A_{j}\right)>0$ and $\left(\mu\left(A_{1}\right), \ldots, \mu\left(A_{k}\right)\right) \in \Delta_{k}$. For any $r>0$, let $N(r)$ be the number of connected components in the coalescence graph $G_{r}$ associated to $A_{1}, \ldots, A_{k}$. The function $(0, \infty) \rightarrow$ $\{1, \ldots, k\}: r \mapsto N(r)$ is non-increasing and right-continuous. Define $r_{i}=\sup \{r>0$ : $N(r) \geq k-i+1\}, i=1, \ldots, k$ and $r_{0}=0$ then it holds

$$
\mu\left(A_{r}\right) \geq 1-(1-\mu(A)) \exp \left(-c \sum_{i=1}^{k} \phi\left(\left[r \wedge r_{i}-r_{i-1}\right]_{+} \sqrt{\lambda^{(k-i+1)}}\right)\right), \forall r>0
$$

where $\phi(x)=\min \left(x ; x^{2}\right), x \geq 0$ and $c$ is the universal constant appearing in Theorem 1.1.

Observe that, contrary to usual concentration results, the bound given above depends on the geometry of the set $A$.

1.3. Proofs. First, we prove Corollary 1.4. The main argument is to repeatedly apply Theorem 1.1 until two sets or more coalesce.

Proof of Corollary 1.4. We proceed by induction over the number of components $k$. For $k=1$, (1.3) follows immediately from Theorem 1.1. Let $k>1$ and let us assume that (1.3) is true for any collection of subsets $B_{1}, \ldots, B_{l}$ satisfying the assumptions of Corollary 1.4 for all $l \in\{1, \ldots, k-1\}$. Let $A_{1}, A_{2}, \ldots, A_{k}$ be a collection of sets satisfying the assumptions of Corollary 1.4. According to Theorem 1.1, it holds

$$
\mu\left(A_{r}\right) \geq 1-(1-\mu(A)) \exp \left(-c \phi\left(r \sqrt{\lambda^{(k)}}\right)\right),
$$

for all $0<r \leq \frac{1}{2} \min _{i \neq j} d\left(A_{i}, A_{j}\right)$.

Let $k_{1}=N\left(\frac{1}{2} \min _{i \neq j} d\left(A_{i}, A_{j}\right)\right)$ and let $i_{1}=k-k_{1}$. Then, for all $i \in\left\{1, \ldots, i_{1}\right\}$, $r_{i}=\frac{1}{2} \min _{i \neq j} d\left(A_{i}, A_{j}\right)$. So that, for all $0<r \leq r_{i_{1}}$, the preceding bound can be 
rewritten as follows (note that only the term of index $i=1$ gives a non zero contribution)

$$
\begin{aligned}
\mu\left(A_{r}\right) & \geq 1-(1-\mu(A)) \exp \left(-c \sum_{i=1}^{i_{1}} \phi\left(\left[r \wedge r_{i}-r_{i-1}\right]_{+} \sqrt{\lambda^{(k-i+1)}}\right)\right) \\
& =1-(1-\mu(A)) \exp \left(-c \sum_{i=1}^{k} \phi\left(\left[r \wedge r_{i}-r_{i-1}\right]_{+} \sqrt{\lambda^{(k-i+1)}}\right)\right)
\end{aligned}
$$

which shows that (1.3) is true for $0<r \leq r_{i_{1}}$. Now let $I_{1}, \ldots, I_{k_{1}}$ be the connected components of $G_{r_{1}}$ and define, for all $i \in\left\{1, \ldots, k_{1}\right\}, B_{i}=\cup_{j \in I_{i}} A_{j, r_{1}}$. It follows easily from Lemma 1.3 that $\left(\mu\left(B_{1}\right), \ldots, \mu\left(B_{k_{1}}\right)\right) \in \Delta_{k_{1}}$. Since $\min _{i \neq j} d\left(B_{i}, B_{j}\right)>0$, the induction hypothesis implies that

$$
\mu\left(B_{s}\right) \geq 1-(1-\mu(B)) \exp \left(-c \sum_{i=1}^{k_{1}} \phi\left(\left[s \wedge s_{i}-s_{i-1}\right]_{+} \sqrt{\lambda^{\left(k_{1}-i+1\right)}}\right)\right), \quad \forall s>0,
$$

where $B=B_{1} \cup \cdots \cup B_{k_{1}}=A_{r_{1}}$ and $s_{i}=\sup \left\{s>0: N^{\prime}(s) \geq k_{1}-i+1\right\}, i \in\left\{1, \ldots, k_{1}\right\}$ $\left(s_{0}=0\right)$ with $N^{\prime}(s)$ the number of connected components in the graph $G_{s}^{\prime}$ associated to $B_{1}, \ldots, B_{k_{1}}$. It is easily seen that $r_{i_{1}+i}=r_{i_{1}}+s_{i}$, for all $i \in\left\{0,1 \ldots, k_{1}\right\}$. Therefore, we have that, for $r>r_{i_{1}}$,

$$
\begin{aligned}
\mu\left(A_{r}\right) & \geq \mu\left(B_{r-r_{i_{1}}}\right) \\
& \geq 1-\left(1-\mu\left(A_{r_{i_{1}}}\right)\right) \exp \left(-c \sum_{i=i_{1}+1}^{k} \phi\left(\left[r \wedge r_{i}-r_{i-1}\right]_{+} \sqrt{\lambda^{(k-i+1)}}\right)\right) \\
& \geq 1-(1-\mu(A)) \exp \left(-c \sum_{i=1}^{k} \phi\left(\left[r \wedge r_{i}-r_{i-1}\right]_{+} \sqrt{\lambda^{(k-i+1)}}\right)\right),
\end{aligned}
$$

where the last line is true by (1.4).

To prove Theorem 1.1, we need some preparatory lemmas. Given a subset $A \subset E$, and $x \in E$, the minimal distance from $x$ to $A$ is denoted by

$$
d(x, A)=\inf _{y \in A} d(x, y) .
$$

Lemma 1.5. Let $A \subset E$ and $\epsilon>0$, then $\left(E \backslash A_{\epsilon}\right)_{\epsilon} \subset E \backslash A$.

Proof. Let $x \in\left(E \backslash A_{\epsilon}\right)_{\epsilon}$. Then, there exists $y \in E \backslash A_{\epsilon}$ (in particular $d(y, A) \geq \epsilon$ ) such that $d(x, y)<\epsilon$. Since the function $z \mapsto d(z, A)$ is 1-Lipschitz, one has

$$
d(x, A) \geq d(y, A)-d(x, y)>0
$$

and so $x \in E \backslash A$.

Remark 1. In fact, we proved that $\left(E \backslash A_{\epsilon}\right)_{\epsilon} \subset E \backslash \bar{A}$. The converse is, in general, not true.

Lemma 1.6. Let $A_{1}, \ldots, A_{k}$ be a family of sets such that $\left(\mu\left(A_{1}\right), \ldots, \mu\left(A_{k}\right)\right) \in \Delta_{k}$ and $r:=\frac{1}{2} \min _{i \neq j} d\left(A_{i}, A_{j}\right)>0$. Let $0<\epsilon \leq r$ and set $A=\cup_{1 \leq i \leq k} A_{i}$ and $A_{0}=E \backslash\left(A_{\epsilon}\right)$. Then,

$$
\max _{i=0, \ldots, k} \frac{\mu\left(A_{i, \epsilon}\right)}{\mu\left(A_{i}\right)} \leq \frac{1-\mu(A)}{1-\mu\left(A_{\epsilon}\right)} .
$$

Proof. First, this is true for $i=0$. Indeed, by definition $A_{0}=E \backslash\left(A_{\epsilon}\right)$ and, according to Lemma 1.5, $\left(A_{0}\right)_{\epsilon} \subset A^{c}$ (the equality is not always true), which proves (1.5) in this 
case. Now, let us show (1.5) for the other values of $i$. Since $\epsilon \leq r$, the $A_{j, \epsilon}$ 's are disjoint sets. Thence, (1.5) is equivalent to

$$
\left(1-\sum_{j=1}^{k} \mu\left(A_{j, \epsilon}\right)\right) \mu\left(A_{i, \epsilon}\right) \leq\left(1-\sum_{j=1}^{k} \mu\left(A_{j}\right)\right) \mu\left(A_{i}\right) .
$$

This inequality is true as soon as

$$
\left(1-\mu\left(A_{i, \epsilon}\right)-m_{i}\right) \mu\left(A_{i, \epsilon}\right) \leq\left(1-\mu\left(A_{i}\right)-m_{i}\right) \mu\left(A_{i}\right),
$$

denoting $m_{i}=\sum_{j \neq i}^{k} \mu\left(A_{j}\right)$. The function $f_{i}(u)=\left(1-u-m_{i}\right) u, u \in[0,1]$, is decreasing on the interval $\left[\left(1-m_{i}\right) / 2,1\right]$. We conclude from this that (1.5) is true for all $i \in$ $\{1, \ldots, k\}$, as soon as $\mu\left(A_{i}\right) \geq\left(1-m_{i}\right) / 2$ for all $i \in\{1, \ldots, k\}$ which amounts to $\left(\mu\left(A_{1}\right), \ldots, \mu\left(A_{k}\right)\right) \in \Delta_{k}$.

For $p>1$, we define the function $\chi_{p}:[0, \infty[\rightarrow[0,1]$ by

$$
\chi_{p}(x)=\left(1-x^{p}\right)^{p}, \quad \text { for } \quad x \in[0,1] \text { and } \quad \chi_{p}(x)=0 \text { for } x>1 .
$$

It is easily seen that $\chi_{p}(0)=1, \chi_{p}^{\prime}(0)=\chi_{p}(1)=\chi_{p}^{\prime}(1)=0$, that $\chi_{p}$ takes values in $[0,1]$ and that $\chi_{p}$ is continuously differentiable on $\left[0, \infty\left[\right.\right.$. We use the function $\chi_{p}$ to construct smooth approximations of indicator functions on $E$, as explained in the next statement.

Lemma 1.7. Let $A \subset E$ and consider the function $f(x)=\chi_{p}(d(x, A) / \epsilon), x \in E$, where $\epsilon>0$ and $p>1$. For all $x \in E$, it holds

$$
|\nabla f|(x) \leq p^{2} \epsilon^{-1} \mathbf{1}_{A_{\epsilon} \backslash A}
$$

Proof. Thanks to the chain rule for the local Lipschitz constant (see e.g. [2, Proposition $2.1])$,

$$
\left|\nabla \chi_{p}\left(\frac{d(\cdot, A)}{\epsilon}\right)\right|(x) \leq \epsilon^{-1} \chi_{p}^{\prime}\left(\frac{d(\cdot, A)}{\epsilon}\right)|\nabla d(\cdot, A)|(x) .
$$

The function $d(\cdot, A)$ being Lipschitz, its local Lipschitz constant is $\leq 1$ and, thereby,

$$
|\nabla f|(x) \leq \chi_{p}^{\prime}\left(\frac{d(x, A)}{\epsilon}\right)
$$

In particular, thanks to the aforementioned properties of $\chi,|\nabla f|$ vanishes on $A$ (and even on $\bar{A}$ ) and on $\{x \in E: d(x, A) \geq \epsilon\}=E \backslash A_{\epsilon}$. On the other hand, a simple calculation shows that $\left|\chi_{p}^{\prime}\right| \leq p^{2}$ which proves the claim.

Proof of Theorem 1.1. Take Borel sets $A_{1}, \ldots, A_{k}$ with $\frac{1}{2} \min _{i \neq j} d\left(A_{i}, A_{j}\right) \geq r>0$ and $\left(\mu\left(A_{1}\right), \ldots, \mu\left(A_{k}\right)\right) \in \Delta_{k}$ and consider $A=A_{1} \cup \cdots \cup A_{k}$. Let us show that, for any $0<\epsilon \leq r$, it holds

$$
\left(1+\lambda^{(k)} \epsilon^{2}\right)\left(1-\mu\left(A_{\epsilon}\right)\right) \leq(1-\mu(A)) .
$$

Let $A_{0}=E \backslash\left(A_{\epsilon}\right)$ and set $f_{i}(x)=\chi_{p}\left(d\left(x, A_{i}\right) / \epsilon\right), x \in E, i \in\{0, \ldots, k\}$, where $p>1$. According to Lemma 1.7 and the fact that $f_{i}=1$ on $A_{i}$, we obtain

$$
\int\left|\nabla f_{i}\right|^{2} \mathrm{~d} \mu=\frac{p^{4}}{\epsilon^{2}} \mu\left(A_{i, \epsilon} \backslash A_{i}\right) \quad \text { and } \quad \int f_{i}^{2} \mathrm{~d} \mu \geq \mu\left(A_{i}\right) .
$$

Since the $f_{i}$ 's have disjoint supports they are orthogonal in $L^{2}(\mu)$ and, in particular, they span a $k+1$ dimensional subspace of $H^{1}(\mu)$. Thus, by definition of $\lambda^{(k)}$,

$$
\lambda^{(k)} \leq \sup _{a \in \mathbb{R}^{k+1}} \frac{\int\left|\nabla\left(\sum_{i=0}^{k} a_{i} f_{i}\right)\right|^{2} \mathrm{~d} \mu}{\int\left(\sum_{i=0}^{k} a_{i} f_{i}\right)^{2} \mathrm{~d} \mu} \leq \sup _{a \in \mathbb{R}^{k+1}} \frac{\int\left(\sum_{i=0}^{k}\left|a_{i}\right|\left|\nabla f_{i}\right|\right)^{2} \mathrm{~d} \mu}{\int\left(\sum_{i=0}^{k} a_{i} f_{i}\right)^{2} \mathrm{~d} \mu},
$$


where the second inequality comes from the following easy to check sub-linearity property of the local Lipschitz constant:

$$
|\nabla(a f+b g)| \leq|a||\nabla f|+|b||\nabla g| .
$$

Since the $f_{i}^{\prime} s$ and the $\left|\nabla f_{i}\right|^{\prime} s$ are two orthogonal families, we conclude using (1.7), that

$$
\frac{\lambda^{(k)} \epsilon^{2}}{p^{4}} \leq \sup _{a \in \mathbb{R}^{k+1}} \frac{\sum_{i=0}^{k} a_{i}^{2}\left(\mu\left(A_{i, \epsilon}\right)-\mu\left(A_{i}\right)\right)}{\sum_{i=0}^{k} a_{i}^{2} \mu\left(A_{i}\right)},
$$

which amounts to

$$
1+\frac{\lambda^{(k)} \epsilon^{2}}{p^{4}} \leq \max _{i=0, \ldots, k} \frac{\mu\left(A_{i, \epsilon}\right)}{\mu\left(A_{i}\right)} .
$$

Applying Lemma 1.6 and sending $p$ to 1 gives (1.6). Now, if $n \in \mathbb{N}$ and $0<\epsilon$ are such that $n \epsilon \leq r$, then iterating (1.6) immediately gives

$$
\left(1+\lambda^{(k)} \epsilon^{2}\right)^{n}\left(1-\mu\left(A_{n \epsilon}\right)\right) \leq 1-\mu(A) .
$$

Optimizing this bound over $n$ for a fixed $\varepsilon$ gives

$$
\left(1-\mu\left(A_{r}\right)\right) \leq(1-\mu(A)) \exp \left(-\sup \left\{\lfloor r / \epsilon\rfloor \log \left(1+\lambda^{(k)} \epsilon^{2}\right): \epsilon \leq r\right\}\right) .
$$

Thus, letting

$$
\Psi(x)=\sup \left\{\lfloor t\rfloor \log \left(1+\frac{x}{t^{2}}\right): t \geq 1\right\}, \quad x \geq 0,
$$

it holds

$$
\left(1-\mu\left(A_{r}\right)\right) \leq(1-\mu(A)) \exp \left(-\Psi\left(\lambda^{(k)} r^{2}\right)\right) .
$$

Using Lemma 1.8 below, we deduce that $\Psi\left(\lambda^{(k)} r^{2}\right) \geq c \min \left(r^{2} \lambda^{(k)} ; r \sqrt{\lambda^{(k)}}\right)$, with $c=$ $\log (5) / 4$, which completes the proof.

Lemma 1.8. The function $\Psi$ defined by (1.9) satisfies

$$
\Psi(x) \geq \frac{\log (5)}{4} \min (x ; \sqrt{x}), \quad \forall x \geq 0 .
$$

Proof. Taking $t=1$, one concludes that $\Psi(x) \geq \log (1+x)$, for all $x \geq 0$. The function $x \mapsto \log (1+x)$ being concave, the function $x \mapsto \frac{\log (1+x)}{x}$ is non-increasing. Therefore, $\log (1+x) \geq \frac{\log (5)}{4} x$ for all $x \in[0,4]$. Now, let us consider the case where $x \geq 4$. Observe that $\lfloor t\rfloor \geq t / 2$ for all $t \geq 1$ and so, for $x \geq 4$,

$$
\Psi(x) \geq \frac{1}{2} \sup _{t \geq 1}\left\{t \log \left(1+\frac{x}{t^{2}}\right)\right\} \geq \frac{\log (5)}{4} \sqrt{x},
$$

by choosing $t=\sqrt{x} / 2 \geq 1$. Thereby,

$$
\Psi(x) \geq \frac{\log (5)}{4}\left[x \mathbf{1}_{[0,4]}(x)+\sqrt{x} \mathbf{1}_{[4, \infty)}(x)\right] \geq \frac{\log (5)}{4} \min (x ; \sqrt{x}),
$$

which completes the proof.

Remark 2. The conclusion of Lemma Lemma 1.8 can be improved. Namely, it can be shown that

$$
\Psi(x)=\max \left(\left(1+\left\lfloor\frac{\sqrt{x}}{a}\right\rfloor\right) \log \left(1+\frac{x}{\left(1+\left\lfloor\frac{\sqrt{x}}{a}\right\rfloor\right)^{2}}\right) ;\left(\left\lfloor\frac{\sqrt{x}}{a}\right\rfloor\right) \log \left(1+\frac{x}{\left(\left\lfloor\frac{\sqrt{x}}{a}\right\rfloor\right)^{2}}\right)\right),
$$


(the second term in the maximum being treated as 0 when $\sqrt{x}<a$ ) where $0<a<2$ is the unique point where the function $(0, \infty) \rightarrow \mathbb{R}: u \mapsto \log \left(1+u^{2}\right) / u$ achieves its supremum. Therefore,

$$
\Psi(x) \sim \frac{\log \left(1+a^{2}\right)}{a} \sqrt{x}
$$

when $x \rightarrow \infty$. The reader can easily check that $\frac{\log \left(1+a^{2}\right)}{a} \simeq 0.8$. In particular, it does not seem possible to reach the constant $c=1$ in Theorem 1.1 using this method of proof.

1.4. Two more multi-set concentration bounds. The condition $\left(\mu\left(A_{1}\right), \ldots, \mu\left(A_{k}\right)\right) \in$ $\Delta_{k}$ can be seen as the multi-set generalization of the condition, standard in concentration of measure, that the size of the enlarged set has to be bigger than $1 / 2$. Indeed, the reader can easily verify that $\left(\frac{1}{k+1}, \ldots, \frac{1}{k+1}\right) \in \Delta_{k}$. However, in practice, this condition can be difficult to check. We provide two more multi-set concentration inequalities that hold in full generality. The method of proof is the same as for Theorem 1.1 and is based on (1.8).

Proposition 1.9. Let $(E, d, \mu)$ be a metric measured space and $\lambda^{(k)}$ be defined as in (1.2). Let $\left(A_{1}, \ldots, A_{k}\right)$ be $k$ Borel sets, $A=\cup_{i} A_{i}$ and $A_{0}=E \backslash A_{r}$. Then, with $a_{(1)}=\min _{1 \leq i \leq k} \mu\left(A_{i}\right)$, the following two bounds hold:

$$
\begin{aligned}
& 1-\mu\left(A_{r}\right) \leq(1-\mu(A)) \frac{1}{\prod_{i=1}^{k} \mu\left(A_{i}\right)} \exp \left(-c \min \left(r^{2} \lambda^{(k)}, r \sqrt{\lambda^{(k)}}\right)\right) ; \\
& 1-\mu\left(A_{r}\right) \leq(1-\mu(A)) \frac{1}{\mu(A)^{\mu(A) / a_{(1)}}} \exp \left(-c \min \left(r^{2} \lambda^{(k)}, r \sqrt{\lambda^{(k)}}\right)\right) .
\end{aligned}
$$

Proof. Fix $N \in \mathbb{N}$ and $\epsilon>0$ such that $N \epsilon \leq r$. For $i=1, \ldots, k$ and $n \leq N$, we define

$$
\begin{aligned}
\alpha_{i}(n) & =\frac{\mu\left(A_{i, n \epsilon}\right)}{\mu\left(A_{i,(n-1) \epsilon}\right)} ; \\
M_{n} & =\max _{1 \leq i \leq k} \alpha_{i}(n) \vee \frac{1-\mu\left(A_{(n-1) \epsilon}\right)}{1-\mu\left(A_{n \epsilon}\right)} ; \\
L_{n} & =\left\{i \in\{1, \ldots, k\} \mid M_{n}=\alpha_{i}(n)\right\} ; \\
N_{i} & =\sharp\left\{n \in\{1, \ldots, N\} \mid i=\inf L_{n}\right\} ; \\
N_{0} & =N-\sum_{i=1}^{k} N_{i} .
\end{aligned}
$$

Roughly speaking, the number $N_{i}(0 \leq i \leq k)$ counts the number of time where the set $A_{i}$ growths in iterating (1.8). Lemma 1.6 asserts that in the case where $\left(\mu\left(A_{1}\right), \ldots, \mu\left(A_{k}\right)\right) \in$ $\Delta_{k}$, then $N_{0}=N$. However, we still obtain from (1.8), for $1 \leq i \leq k$,

$$
\frac{1}{\mu\left(A_{i}\right)} \geq \prod_{n=1}^{N} \alpha_{i}(n) \geq\left(1+\lambda^{(k)} \epsilon^{2}\right)^{N_{i}}
$$

The first inequality is true because $\mu\left(A_{i, N \epsilon}\right) \leq 1$ and a telescoping argument. The second inequality is true because, as $n$ ranges from 1 to $N$, by definition of the number $N_{i}$ and (1.8), there are, at least $N_{i}$ terms appearing in the product that can be bounded by $\left(1+\lambda^{(k)} \epsilon^{2}\right)$. The other terms are bounded above by 1 . The case of $i=0$ is handled in a similar fashion and we obtain:

$$
\begin{aligned}
1-\mu\left(A_{N \epsilon}\right) & \leq(1-\mu(A))\left(1+\lambda^{(k)} \epsilon^{2}\right)^{-N_{0}} \\
& =(1-\mu(A))\left(1+\lambda^{(k)} \epsilon^{2}\right)^{-N} \prod_{i=1}^{k}\left(1+\lambda^{(k)} \epsilon^{2}\right)^{N_{i}} .
\end{aligned}
$$


The announced bounds will be obtain by bounding the product appearing in the righthand side and an argument similar to the end of the proof of Theorem 1.1. From (1.10), we have that,

$$
\prod_{i=1}^{k}\left(1+\lambda^{(k)} \epsilon^{2}\right)^{N_{i}} \leq \frac{1}{\prod_{i=1}^{k} \mu\left(A_{i}\right)} .
$$

Also, from (1.10),

$$
\mu\left(A_{i, N \epsilon}\right) \geq\left(1+\lambda^{(k)} \epsilon^{2}\right)^{N_{i}} \mu\left(A_{i}\right) .
$$

Because $N \epsilon \leq r$, the sets $A_{1, N \epsilon}, \ldots, A_{k, N \epsilon}$ are pairwise disjoint and, thereby,

$$
1 \geq \sum \mu\left(A_{i, N \epsilon}\right) \geq \sum_{i=1}^{k}\left(1+\lambda^{(k)} \epsilon^{2}\right)^{N_{i}} \mu\left(A_{i}\right)
$$

Fix $\theta>0$ to be chosen later. By convexity of exp,

$$
\begin{aligned}
1+(1-\mu(A))\left(1+\lambda^{(k)} \epsilon^{2}\right)^{\theta} & \geq \exp \left(\left(\sum_{i=1}^{k} \mu\left(A_{i}\right) N_{i}+(1-\mu(A)) \theta\right) \log \left(1+\lambda^{(k)} \epsilon^{2}\right)\right) \\
& \geq \exp \left(\left(a_{(1)} \sum_{i=1}^{k} N_{i}+(1-\mu(A)) \theta\right) \log \left(1+\lambda^{(k)} \epsilon^{2}\right)\right) .
\end{aligned}
$$

Finally, with $p=1-\mu(A)$ and $t=\theta \log \left(1+\lambda^{(k)} \epsilon^{2}\right)$, we obtain

$$
\prod_{i=1}^{k}\left(1+\lambda^{(k)} \epsilon^{2}\right)^{N_{i}} \leq\left(\mathrm{e}^{-p t}+p \mathrm{e}^{(1-p) t}\right)^{1 / a_{(1)}} .
$$

We easily check that, the quantity in the right-hand side is minimal for $t=\log \frac{1}{1-p}$ at which it takes the value $(1-p)^{p-1}=\mu(A)^{-\mu(A) / a_{(1)}}$. Thus,

$$
\prod_{i=1}^{k}\left(1+\lambda^{(k)} \epsilon^{2}\right)^{N_{i}} \leq \frac{1}{\mu(A)^{\mu(A) / a_{(1)}}} .
$$

Combining (1.12) and (1.13) with (1.11) and the same argument as for (1.9), we obtain the two announced bounds.

From Proposition 1.9, we can derive bounds on the $\lambda^{(k)}$ 's. The proof is the same as the one of Proposition 1.2 and is omitted.

Proposition 1.10. Let $(E, d, \mu)$ be a metric measured space and $\lambda^{(k)}$ be defined as in (1.2). Let $A_{1}, \ldots, A_{k}$ be measurable sets, then, with $r=\frac{1}{2} \min _{i \neq j} d\left(A_{i}, A_{j}\right)$ and $A_{0}=E \backslash\left(\cup A_{i}\right)_{r}$,

$$
\begin{aligned}
\lambda^{(k)} & \leq \frac{1}{r^{2}} \psi\left(\frac{1}{c} \ln \frac{a_{(1)}}{\mu\left(A_{0}\right)}+\frac{1}{c} k \ln \frac{1}{a_{(1)}}\right) ; \\
\lambda^{(k)} & \leq \frac{1}{r^{2}} \psi\left(\frac{1}{c} \ln \frac{a_{(1)}}{\mu\left(A_{0}\right)}+\frac{1}{c} \frac{\mu(A)}{a_{(1)}} \ln \frac{1}{\mu(A)}\right),
\end{aligned}
$$

where $\psi(x)=\max \left(x, x^{2}\right)$ and $a_{(1)}=\min _{1 \leq i \leq k} \mu\left(A_{i}\right)$. 
1.5. Comparison with the result of Chung-Grigor'yan-Yau. In [11], the authors obtained the following result:

Theorem 1.11 (Chung-Grigoryan-Yau [11]). Let $M$ be a compact connected smooth Riemannian manifold equipped with its geodesic distance $d$ and normalized Riemannian volume $\mu$. For any $k \geq 1$ and any family of sets $A_{0}, \ldots, A_{k}$, it holds

$$
\lambda^{(k)} \leq \frac{1}{\min _{i \neq j} d^{2}\left(A_{i}, A_{j}\right)} \max _{i \neq j} \log \left(\frac{4}{\mu\left(A_{i}\right) \mu\left(A_{j}\right)}\right)^{2},
$$

where $1=\lambda^{(0)} \leq \lambda^{(1)} \leq \cdots \lambda^{(k)} \leq \cdots$ denotes the discrete spectrum of $-\Delta$.

Let us translate this result in terms of concentration of measure. Let $A_{1}, \ldots, A_{k}$ be sets such that $r=\frac{1}{2} \min _{1 \leq i<j \leq k} d\left(A_{i}, A_{j}\right)>0$ and define $A=A_{1} \cup \cdots \cup A_{k}$ and $A_{0}=M \backslash A_{s}$, for some $0<s \leq r$. Then, applying (1.14) to this family of $k+1$ sets gives the following inequality

$$
\min \left(a_{(2)} ; 1-\mu\left(A_{s}\right)\right) \leq \frac{4}{a_{(1)}} \exp \left(-\sqrt{\lambda^{(k)}} s\right)
$$

with $a_{(1)}$ and $a_{(2)}$ being respectively the smallest number and the second smallest number among $\left(\mu\left(A_{1}\right), \ldots, \mu\left(A_{k}\right)\right)$ (counted with multiplicity). Note that the right hand side is less than or equal to $a_{(2)}$ if and only if $s \geq s_{o}:=\frac{1}{\sqrt{\lambda^{(k)}}} \log \left(\frac{4}{a_{(1)} a_{(2)}}\right)$, so that (1.15) is equivalent to the following statement:

$$
\mu\left(A_{s}\right) \geq 1-\frac{4}{a_{(1)}} \exp \left(-\sqrt{\lambda^{(k)}} s\right), \quad \forall s \in\left[\min \left(s_{o}, r\right) ; r\right] .
$$

We note that (1.16) holds for any family of sets, whereas the inequality given in Theorem 1.1 is only true when $\left(\mu\left(A_{1}\right), \ldots, \mu\left(A_{k}\right)\right) \in \Delta_{k}$. Also due to the fact that the constant $c$ appearing in Theorem 1.1 is less than 1, (1.16) is asymptotically better than ours (see also Remark 2 above). On the other hand, one sees that (1.16) is only valid for $s$ large enough (and its domain of validity can thus be empty when $s_{o}>r$ ) whereas our inequality is true on the whole interval $(0, r]$. It does not seem also possible to iterate (1.16) as we did in Corollary 1.4. Finally, observe that the method of proof used in [11] and [10] is based on heat kernel bounds and is very different from ours.

Let us translate Theorem 1.11 in a form closer to our Proposition 1.2. Fix $k$ sets $A_{1}, \ldots, A_{k}$ such that $\left(\mu\left(A_{1}\right), \ldots, \mu\left(A_{k}\right)\right) \in \Delta_{k}$. Let $2 r=\min d\left(A_{i}, A_{j}\right)$, where the infimum runs on $i, j=1, \ldots, k$ with $i \neq j$. We have to choose a $(k+1)$-th set. In view of Theorem 1.11, the most optimal choice is to choose $A_{0}=E \backslash\left(\cup A_{i}\right)_{r}$. Indeed, it is the biggest set (in the sense of inclusion) such that $\min d\left(A_{i}, A_{j}\right)=r$ where this time the infimum runs on $i, j=0, \ldots, k$ and $i \neq j$. We let $a_{(0)}=\mu\left(A_{0}\right)$ and we remark that if $\left(\mu\left(A_{1}\right), \ldots, \mu\left(A_{k}\right)\right) \in \Delta_{k}$ then $a_{(0)} \leq a_{(1)}$. The bound (1.14) can be read: for all $r>0$,

$$
\lambda^{(k)} \leq \frac{1}{r^{2}}\left(\log \frac{4}{a_{(1)} a_{(0)}}\right)^{2} .
$$

Therefore, to compare it to our bound, we need to solve

$$
\phi^{-1}\left(\frac{1}{c} \log \frac{a_{(1)}}{a_{(0)}}\right)^{2} \leq\left(\log \frac{4}{a_{(1)} a_{(0)}}\right)^{2} .
$$

Because the right-hand side is always $\geq 1$, taking the square root and composing with the non-decreasing function $\phi$ yields

$$
\frac{1}{c} \log \frac{a_{(1)}}{a_{(0)}} \leq \log \frac{4}{a_{(1)} a_{(0)}} .
$$


That is

$$
a_{(1)}^{1+c} \leq 4^{c} a_{(0)}^{1-c} .
$$

In other words, on some range our bound is better and in some other range their bound is better. However, if the constant $c=1$ could be attained in Theorem 1.1, this would show that our bound is always better. Note that comparing the bounds obtained in Proposition 1.10 and the one of [11] is not so clear as, without the assumption that $\left(\mu\left(A_{1}\right), \ldots, \mu\left(A_{k}\right)\right) \in \Delta_{k}$ it is not necessary that $a_{(0)} \leq a_{(1)}$ and in that case we would have to compare different sets.

\section{Eigenvalue estimates for NON-NEGATIVEly CURVED SPACES}

We recall the values of the $\lambda^{(k)}$ 's that appear in Theorem 1.1 in the case of two important models of positively curved spaces in geometry. Namely:

(i) The $n$-dimensional sphere of radius $\sqrt{\frac{n-1}{\rho}}, \mathbb{S}^{n, \rho}$ endowed with the natural geodesic distance $d_{n, \rho}$ arising from its canonical Riemannian metric and its normalized volume measure $\mu_{n, \rho}$ which has constant Ricci curvature equals to $\rho$ and dimension $n$.

(ii) The $n$-dimensional Euclidean space $\mathbb{R}^{n}$ endowed with the $n$-dimensional Gaussian measure of covariance $\rho^{-1} \mathrm{Id}$,

$$
\gamma_{n, \rho}(\mathrm{d} x)=\frac{\rho^{n / 2} \mathrm{e}^{-\rho|x|^{2} / 2}}{(2 \pi)^{n / 2}} \mathrm{~d} x .
$$

This space has dimension $\infty$ and curvature bounded below by $\rho$ in the sense of [4].

These models arise as weighted Riemannian manifolds without boundary having a purely discrete spectrum. In that case, it was proved in [23, Proposition 3.2] that the $\lambda_{k}$ 's of (1.2) are exactly the eigenvalues (counted with multiplicity) of a self-adjoint operator that we give explicitly in the following. Using a comparison between eigenvalues of [23], we obtain an estimates for eigenvalues in the case of log-concave probability measure over the Euclidean $\mathbb{R}^{n}$.

Example 1 (Spheres). On $\mathbb{S}^{n, \rho}$, the eigenvalues of minus the Laplace-Beltrami operator (see for instance [3, Chapter 3]) are of the form $\rho^{-2}(n-1)^{2} l(l+n-1)$ for $l \in \mathbb{N}$ and the dimension of the corresponding eigenspace $H_{l, n}$ is

$$
\operatorname{dim} H_{l, n}=\frac{2 l+n-1}{l}\left(\begin{array}{c}
l+n-2 \\
l-1
\end{array}\right), \text { if } l>0 \text { and } \operatorname{dim} H_{l, n}=1, \text { if } l=0 .
$$

Consequently,

$$
D_{l, n}:=\operatorname{dim} \bigoplus_{l^{\prime}=0}^{l} H_{l^{\prime}, n}=\left(\begin{array}{c}
n+l \\
l
\end{array}\right)+\left(\begin{array}{c}
n+l-1 \\
l-1
\end{array}\right),
$$

and $\lambda^{(k)}=\rho^{-2}(n-1)^{2} l(l+n-1)$ if and only if $D_{l-1, n}<k \leq D_{l, n}$ where $\lambda^{(k)}$ is the $k$-th eigenvalues of $-\Delta_{\mathbb{S} n, \rho}$ and coincides with the variational definition given in (1.2).

Example 2 (Gaussian spaces). On the Euclidean space $\mathbb{R}^{n}$, equipped with the Gaussian measure $\gamma_{n, \rho}$, the corresponding weighted Laplacian is $\Delta_{\gamma_{n, \rho}}=\Delta_{\mathbb{R}^{n}}-\rho x \cdot \nabla$. The eigenvalues of $-\Delta_{\gamma_{n, \rho}}$ are exactly of the form $\rho^{2} q$ and the dimension of the associated eigenspace $H_{q, n}$ is

$$
\operatorname{dim} H_{q, n}=\left(\begin{array}{c}
n+q-1 \\
q
\end{array}\right)
$$


Consequently,

$$
D_{q, n}:=\operatorname{dim} \bigoplus_{q^{\prime}=0}^{q} H_{q^{\prime}, n}=\left(\begin{array}{c}
n+q \\
q
\end{array}\right),
$$

and $\lambda^{(k)}=\rho^{-2} q$ if and only if $D_{q-1, n}<k \leq D_{q, n}$ where $\lambda^{(k)}$ is the $k$-th eigenvalues of $-\Delta_{\gamma_{n, \rho}}$ and coincides with the variational definition given in (1.2).

Example 3 (Log-concave Euclidean spaces). We study the case where $E=\mathbb{R}^{n}, d$ is the Euclidean distance and $\mu$ is a strictly log-concave probability measure. By this we mean that $\mu(\mathrm{d} x)=\mathrm{e}^{-V(x)} \mathrm{d} x$, where $V: \mathbb{R}^{n} \rightarrow \mathbb{R}$ such that $V$ is $\mathcal{C}^{2}$ and satisfying $\nabla^{2} V \geq K$ for some $K>0$. It is a consequence of [4, Proposition 4] that such a condition on $V$ implies that the semigroup generated by the solution of the stochastic differential equation

$$
\mathrm{d} X_{t}=\sqrt{2} \mathrm{~d} B_{t}-\nabla V\left(X_{t}\right) \mathrm{d} t
$$

where $B$ is a Brownian motion on $\mathbb{R}^{n}$, satisfies the curvature-dimension $C D(\infty, K)$ of Bakry-Emery and, therefore, holds the log-Sobolev inequality, for all $f \in \mathcal{C}_{c}^{\infty}\left(\mathbb{R}^{n}\right)$,

$$
\text { Ent }_{\mu} f^{2} \leq \frac{2}{K} \int|\nabla f(x)|^{2} \mu(\mathrm{d} x) .
$$

Such an inequality implies the super-Poincaré of [27, Theorem 2.1] that in turns implies that the self-adjoint operator $L=-\Delta+\nabla V \cdot \nabla$ has a purely discrete spectrum. In that case, the $\lambda^{(k)}$ of (1.2) corresponds to these eigenvalues and [23] showed that

$$
\lambda^{(k)} \geq \lambda_{\gamma_{n, \rho}}^{(k)}
$$

where $\lambda_{\gamma_{n, \rho}}^{(k)}$ is the eigenvalues of $-\Delta_{\gamma_{n, \rho}}$ of the previous example.

\section{Extension to Markov chains}

As in the classical case (see [19, Theorem 3.3]), our continuous result admits a generalization on finite graphs or more broadly in the setting of Markov chains on a finite state space. We consider a finite set $E$ and $X=\left(X_{n}\right)_{n \in \mathbb{N}}$ be a irreducible time-homogeneous Markov chain with state space $E$. We write $p(x, y)=\mathbb{P}\left(X_{1}=y \mid X_{0}=x\right)$ and we regard $p$ as a matrix. We assume that $p$ admits a reversible probability measure $\mu$ on $E$ : $p(x, y) \mu(x)=p(y, x) \mu(y)$ for all $x, y \in E$ (which implies in particular that $\mu$ is invariant). The Markov kernel $p$ induces a graph structure on $E$ by the following procedure. Set the elements of $E$ as the vertex of the graph and for $x, y \in E$ connect them with an edge if $p(x, y)>0$. As the chain is irreducible, this graph is connected. We equip $E$ with the induced graph distance $d$. We write $L=p-I$, where $I$ stands for the identity matrix. The operator $-L$ is a symmetric positive operator on $\mathcal{L}^{2}(\mu)$. We let $\lambda^{(k)}$ be the eigenvalues of this operator. Then, our Theorem 1.1 extends as follows:

Theorem 3.1. For any $k \geq 1$ and for all sets $A_{1}, \ldots, A_{k} \subset E$ such that $\min _{i \neq j} d\left(A_{i}, A_{j}\right) \geq$ 1 and $\left(\mu\left(A_{1}\right), \ldots, \mu\left(A_{k}\right)\right) \in \Delta_{k}$ the set $B=A_{1} \cup A_{2} \cup \cdots \cup A_{k}$ satisfies

$$
\mu\left(B_{n}\right) \geq 1-(1-\mu(B))\left(1+\lambda^{(k)}\right)^{-n},
$$

for all $1 \leq n \leq \frac{1}{2} \min _{i \neq j} d\left(A_{i}, A_{j}\right)$ where $\lambda^{(k)}$ is the $k$-th eigenvalue of the operator $-L$ acting on $\mathcal{L}^{2}(\mu)$.

Proof. We let $\Pi(x, y)=p(x, y) \mu(x)$ and

$$
\mathscr{E}(f, g)=\frac{1}{2} \sum(f(y)-f(x))(g(y)-g(x)) \Pi(x, y)=\langle f,-L g\rangle_{\mu} .
$$

For any set $A$, we define the discrete boundary of $A$ as $\partial A=A_{1} \backslash A \cup\left(A^{C}\right)_{1} \backslash A^{C}$. Let $\left(X_{n}\right)$ be the Markov chain with transition kernel $p$ and initial distribution $\mu$. By 
reversibility of $\mu,\left(X_{0}, X_{1}\right)$ is an exchangeable pair of law $\Pi$ whose the marginals are given by $\mu$. Then, for a set $U$, we have

$$
\mathscr{E}\left(1_{U}\right)=\mathbb{E} 1_{U}\left(X_{0}\right)\left(1_{U}\left(X_{0}\right)-1_{U}\left(X_{1}\right)\right)=\mathbb{P}\left(X_{0} \in U, X_{1} \notin U\right) \leq \mathbb{P}\left(X_{1} \in \partial U\right)=\mu(\partial U) .
$$

Observe that if $d(U, V) \geq 1, U$ and $V$ are disjoint and $U \times V \notin \operatorname{supp} \Pi$ so that $\mathscr{E}\left(1_{U}, 1_{V}\right)=0$. By Courant-Fischer's min-max theorem

$$
\lambda^{(k)}=\min _{\operatorname{dim} V=k+1} \max _{f \in V} \frac{\mathscr{E}(f, f)}{\mu\left(f^{2}\right)} .
$$

Choose sets $A_{1}, \ldots, A_{k}$ with $d\left(A_{i}, A_{j}\right) \geq 2 n(i \neq j)$ and $\left(\mu\left(A_{1}\right), \ldots, \mu\left(A_{k}\right)\right) \in \Delta_{k}$. Set $f_{i}=1_{A_{i}}$. The $f_{i}$ 's have disjoint support and so they are orthogonal in $L^{2}(\mu)$. By the previous variational representation of $\lambda^{(k)}$, we have

$$
\lambda^{(k)} \leq \sup _{a_{i}} \frac{\mathscr{E}\left(\sum_{i=0}^{k} a_{i} f_{i}\right)}{\int\left(\sum_{i=0}^{k} a_{i} f_{i}\right)^{2} \mathrm{~d} \mu}=\sup _{a_{i}} \frac{\sum a_{i} a_{i^{\prime}} \mathscr{E}\left(f_{i}, f_{i^{\prime}}\right)}{\sum a_{i} a_{i^{\prime}} \int f_{i} f_{i^{\prime}} \mathrm{d} \mu}=\sup _{a_{i}} \frac{\sum_{i=0}^{k} a_{i}^{2} \mathscr{E}\left(f_{i}\right)}{\sum_{i=0}^{k} a_{i} \int f_{i}^{2} \mathrm{~d} \mu} .
$$

In other words,

$$
\lambda^{(k)} \leq \max _{i=0, \ldots, k} \frac{\mu\left(\left(A_{i}\right)_{1}\right)+\mu\left(\left(A_{i}^{C}\right)_{1}\right)-1}{\mu\left(A_{i}\right)} \leq \frac{\mu\left(\left(A_{i}\right)_{1}\right)-\mu\left(A_{i}\right)}{\mu\left(A_{i}\right)},
$$

where the last inequality comes from the fact that, by Lemma 1.5, $\mu\left(E \backslash(E \backslash A)_{1}\right) \geq$ $\mu(A)$. Consider the set $B=\cup_{i=1}^{k} A_{i}$ and choose $A_{0}=E \backslash B_{1}$. In that case, by Lemma 1.6 with $\epsilon=1$, we have

$$
\max _{i=0, \ldots, k} \frac{\mu\left(\left(A_{i}\right)_{1}\right)}{\mu\left(A_{i}\right)} \leq \frac{1-\mu(B)}{1-\mu\left(B_{1}\right)} .
$$

Thus, we proved that

$$
\left(1+\lambda^{(k)}\right)\left(1-\mu\left(B_{1}\right)\right) \leq(1-\mu(B)) .
$$

We derive the announced result by an immediate recursion.

\section{Functional Forms of the MUltiple SEtS CONCENTRATion PROPERTy}

We investigate the functional form of the multi-sets concentration of measure phenomenon results obtained in Sections 1 and 3.

Proposition 4.1. Let $(E, d)$ be a metric space equipped with a Borel probability measure $\mu$. Let $\alpha_{k}:[0, \infty) \rightarrow[0, \infty)$. The following properties are equivalent:

(1) For all Borel sets $A_{1}, \ldots, A_{k} \subset E$ such that $\left(\mu\left(A_{1}\right), \ldots, \mu\left(A_{k}\right)\right) \in \Delta_{k}$, the set $A=A_{1} \cup \cdots \cup A_{k}$ satisfies

$$
\mu\left(A_{r}\right) \geq 1-(1-\mu(A)) \alpha_{k}(r), \quad \forall 0<r \leq \frac{1}{2} \min _{i \neq j} d\left(A_{i}, A_{j}\right) .
$$

(2) For all 1-Lipschitz functions $f_{1}, \ldots, f_{k}: E \rightarrow \mathbb{R}$ such that the sublevel sets $A_{i}=\left\{f_{i} \leq 0\right\}$ are such that $\left(\mu\left(A_{1}\right), \ldots, \mu\left(A_{k}\right)\right) \in \Delta_{k}$, the function $f^{*}=$ $\min \left(f_{1}, \ldots, f_{k}\right)$ satisfies

$$
\mu\left(f^{*}<r\right) \geq 1-\mu\left(f^{*} \leq 0\right) \alpha_{k}(r), \quad \forall 0<r \leq \frac{1}{2} \min _{i \neq j} d\left(A_{i}, A_{j}\right) .
$$

Together with Theorem 1.1 or Theorem 3.1, one thus sees that the presence of multiple wells can improve the concentration properties of a Lipschitz function. 
Proof. It is clear that (2) implies (1) when applied to $f_{i}(x)=d\left(x, A_{i}\right)$, in which case $A_{i}=\left\{f_{i} \leq 0\right\}$ and $f^{*}(x)=d(x, A)$. The converse is also very classical. First, observe that $\left\{f^{*}<r\right\}=\cup_{i=1}^{k}\left\{f_{i}<r\right\}$. Then, since $f_{i}$ is 1-Lipschitz, it holds $A_{i, r} \subset\left\{f_{i}<r\right\}$ with $A_{i}=\left\{f_{i} \leq 0\right\}$ and so letting $A=A_{1} \cup \cdots \cup A_{k}$, it holds $A_{r} \subset\left\{f^{*}<r\right\}$. Therefore, applying (1) to this set $A$ gives (2).

When (4.1) holds, we will say that the probability metric space $(E, d, \mu)$ satisfies the multi-set concentration of measure property of order $k$ with the concentration profile $\alpha_{k}$.

In the usual setting $(k=1)$, the concentration of measure phenomenon implies deviation inequalities for Lipschitz functions around their median. The next result generalizes this well known fact to $k>1$.

Proposition 4.2. Let $(E, d, \mu)$ be a probability metric space satisfying the multi-set concentration of measure property of order $k$ with the concentration profile $\alpha_{k}$ and $f$ : $E \rightarrow \mathbb{R}$ be a 1-Lipschitz function. If $I_{1}, \ldots, I_{k} \subset \mathbb{R}$ are $k$ disjoint Borel sets such that $\left(\mu\left(f \in I_{1}\right), \ldots, \mu\left(f \in I_{k}\right)\right) \in \Delta_{k}$, then it holds

$$
\mu\left(f \in \cup_{i=1}^{k} I_{i, r}\right) \geq 1-\left(1-\mu\left(f \in \cup_{i=1}^{k} I_{i}\right)\right) \alpha_{k}(r), \quad \forall 0<r \leq \frac{1}{2} \min _{i \neq j} d\left(I_{i}, I_{j}\right)
$$

Proof. Let $\nu$ be the image of $\mu$ under the map $f$. Since $f$ is 1-Lipschitz, the metric space $(\mathbb{R},|\cdot|, \nu)$ satisfies the multi-set concentration of measure property of order $k$ with the same concentration profile $\alpha_{k}$ as $\mu$. Details are left to the reader.

Let us conclude this section by detailing an application of potential interest in approximation theory.

Suppose that $f: E \rightarrow \mathbb{R}$ is some 1-Lipschitz function and $A_{1}, \ldots, A_{k}$ are (pairwise disjoint) subsets of $E$ such that $\left(\mu\left(A_{1}\right), \ldots, \mu\left(A_{k}\right)\right) \in \Delta_{k}$. Let us assume that the restrictions $f_{\mid A_{i}}, i \in\{1, \ldots, k\}$ are known and that one wishes to estimate or reconstruct $f$ outside $A=\cup_{i=1}^{k} A_{i}$. To that aim, one can consider an explicit 1-Lipschitz extension of $f_{\mid A}$, that is to say a 1-Lipschitz function $g: E \rightarrow \mathbb{R}$ (constructed based on our knowledge of $f$ on $A$ exclusively) such that $f=g$ on $A$. There are several canonical ways to perform the extension of a Lipschitz function defined on a sub domain (known as Kirszbraun-McShane-Whitney extensions [18, 22, 28]). One can consider for instance the functions

$$
g_{+}(x)=\inf _{y \in A}\{f(y)+d(x, y)\} \quad \text { or } \quad g_{-}(x)=\sup _{y \in A}\{f(y)-d(x, y)\}, \quad x \in E .
$$

It is a very classical fact that functions $g_{-}$and $g_{+}$are 1-Lipschitz extensions of $f_{\mid A}$ and moreover that any extension $g$ of $f_{\mid A}$ satisfies $g_{-} \leq g \leq g_{+}$(see $e . g$ [17]).

The following simple result shows that, for any 1-Lipschitz extension $g$ of $f_{\mid A}$, the probability of error $\mu(|f-g|>r)$ is controlled by the multi-set concentration profile $\alpha_{k}$. In particular, in the framework of our Theorem 1.1, this probability of error is expressed in terms of $\lambda^{(k)}$.

Proposition 4.3. Let $(E, d, \mu)$ be a probability metric space satisfying the multi-set concentration of measure property of order $k$ with the concentration profile $\alpha_{k}$ and $f: E \rightarrow \mathbb{R}$ be a 1-Lipschitz function. Let $A_{1}, \ldots A_{k}$ be subsets of $E$ such that $\left(\mu\left(A_{1}\right), \ldots, \mu\left(A_{k}\right)\right) \in$ $\Delta_{k}$; then for any 1-Lipschitz extension $g$ of $f_{\mid A}$, it holds

$$
\mu(|f-g| \geq r) \leq(1-\mu(A)) \alpha_{k}(r / 2), \quad \forall 0<r \leq \min _{i \neq j} d\left(A_{i}, A_{j}\right) .
$$

Proof. The function $h: E \rightarrow \mathbb{R}$ defined by $h(x)=|f-g|(x), x \in E$, is 2-Lipschitz and vanishes on $A$. Therefore, for any $x \in E$ and $y \in A$, it holds $h(x) \leq h(y)+$ 
$2 d(x, y)=2 d(x, y)$. Optimizing over $y \in A$ gives that $h(x) \leq 2 d(x, A)$. Therefore $\{h \geq r\} \subset\{x: d(x, A) \geq r / 2\}=\left(A_{r / 2}\right)^{c}$ and so, if $0<r \leq \min _{i \neq j} d\left(A_{i}, A_{j}\right)$, it holds

$$
\mu(|f-g| \geq r) \leq(1-\mu(A)) \alpha_{k}(r / 2) .
$$

Remark 3. Let us remark that Propositions 4.1 to 4.3 can be immediately extended under the following more general (but notationally heavier) multi-set concentration of measure assumption: there exists functions $\alpha_{k}:[0, \infty) \rightarrow[0, \infty)$ and $\beta_{k}:[0, \infty)^{k} \rightarrow$ $[0, \infty]$ such that for all Borel sets $A_{1}, \ldots, A_{k} \subset E$, the set $A=A_{1} \cup \cdots \cup A_{k}$ satisfies

$$
\mu\left(A_{r}\right) \geq 1-\beta_{k}\left(\mu\left(A_{1}\right), \cdots, \mu\left(A_{k}\right)\right) \alpha_{k}(r), \quad \forall 0<r \leq \frac{1}{2} \min _{i \neq j} d\left(A_{i}, A_{j}\right) .
$$

This framework contains the preceding one, by choosing $\beta_{k}(a)=1-\sum_{i=1}^{k} a_{i}$ if $a=$ $\left(a_{1}, \ldots, a_{k}\right) \in \Delta_{k}$ and $+\infty$ otherwise. It also contains the concentration bounds obtained in Proposition 1.9, corresponding respectively to

$$
\beta_{k}(a)=\frac{1-\sum_{i=1}^{k} a_{i}}{\prod_{i=1}^{k} a_{i}}, \text { and } \beta_{k}(a)=\frac{1-\sum_{i=1}^{k} a_{i}}{\left(\sum_{i=1}^{k} a_{i}\right)^{\sum_{i=1}^{k} a_{i} / \min \left(a_{1}, \cdots, a_{k}\right)}}, \quad a=\left(a_{1}, \ldots, a_{k}\right) .
$$

\section{Open questions}

We list open questions related to the multi-set concentration of measure phenomenon.

5.1. Gaussian multi-set concentration. Using the terminology introduced in Section 4, Theorem 1.1 and the material exposed in Section 2 tell us that, if $\mu$ has a density of the form $e^{-V}$ with respect to Lebesgue measure on $\mathbb{R}^{n}$ with a smooth function $V$ such that Hess $V \geq \rho>0$, then the probability metric space $\left(\mathbb{R}^{n},|\cdot|, \mu\right)$ satisfies the multi-set concentration of measure property of order $k$ with the concentration profile

$$
\alpha_{k}(r)=\exp \left(-c \min \left(r^{2} \lambda_{\gamma_{n}, \rho}^{(k)} ; r \sqrt{\left.\lambda_{\gamma_{n}, \rho}^{(k)}\right)}\right), \quad r \geq 0,\right.
$$

where $\lambda_{\gamma_{n}, \rho}^{(k)}$ denotes the $k$ th eigenvalue of the $n$-dimensional centered Gaussian measure with covariance matrix $\rho^{-1} \mathrm{Id}$. Since the measure $\mu$ satisfies the log-Sobolev inequality, it is well known that it satisfies a (classical) Gaussian concentration of measure inequality. Therefore, it is natural to conjecture that $\mu$ satisfies a multi-set concentration of measure property of order $k \geq 1$ with a profile of the form

$$
\beta_{k}(r)=\exp \left(-C_{k, \rho, n} r^{2}\right), \quad r \geq 0,
$$

for some constant $C_{k, \rho, n}$ depending solely on its arguments. In addition, it would be interesting to see how usual functional inequalities (Log-Sobolev, transport-entropy, ....) can be modified to catch such a concentration of measure phenomenon.

5.2. Equivalence between multi-set concentration and lower bounds on eigenvalues in non-negative curvature. Let us quickly recall the main finding of E. Milman $[24,25]$, that is, under non-negative curvature assumptions, a concentration of measure estimate implies a bound on the spectral gap. Let $\mu$ be a probability measure with a density of the form $e^{-V}$ on a smooth connected Riemannian manifold $M$ with $V$ a smooth function such that

$$
\text { Ric }+ \text { Hess } V \geq 0 \text {. }
$$

Assume that $\mu$ satisfies a concentration inequality of the form: for all $A \subset M$ such that $\mu(A) \geq 1 / 2$

$$
\mu\left(A_{r}\right) \geq 1-\alpha(r), \quad r \geq 0
$$


where $\alpha$ is a function such that $\alpha\left(r_{o}\right)<1 / 2$ for at least one value $r_{o}>0$. Then, letting $\lambda^{(1)}$ be the first non zero eigenvalue of the operator $-\Delta+\nabla V \cdot \nabla$, it holds $\lambda^{(1)} \geq \frac{1}{4}\left(\frac{1-2 \alpha\left(r_{o}\right)}{r_{o}}\right)^{2}$. It would be very interesting to extend Milman's result to a multiset concentration setting. More precisely, if $\mu$ satisfies the curvature condition (5.1) and the multi-set concentration of measure property of order $k$ with a profile of the form $\alpha_{k}(r)=\exp \left(-\min \left(a r^{2}, \sqrt{a} r\right)\right), r \geq 0$, can we find a universal function $\varphi_{k}$ such that $\lambda^{(k)} \geq \varphi_{k}(a) ?$

This question already received some attention in recent works by Funano and Shioya $[13,14]$. In particular, let us mention the following improvement of the ChungGrigor'yan-Yau inequality obtained in [13]. There exists a universal constant $c>1$ such that if $\mu$ is a probability measure satisfying the non-negative curvature assumption (5.1), it holds: for any family of sets $A_{0}, A_{1}, \ldots, A_{l}$ with $1 \leq l \leq k$

$$
\lambda^{(k)} \leq c^{k-l+1} \frac{1}{\min _{i \neq j} d^{2}\left(A_{i}, A_{j}\right)} \max _{i \neq j} \log \left(\frac{4}{\mu\left(A_{i}\right) \mu\left(A_{j}\right)}\right)^{2} .
$$

Note that the difference with (1.14) is that $\lambda^{(k)}$ is estimated by a reduced number of sets. Using (5.2) (with $l=1$ ) together with Milman's result recalled above, Funano showed that there exists some constant $C_{k}$ depending only on $k$ such that under the curvature condition (5.1), it holds $\lambda^{(k)} \leq C_{k} \lambda^{(1)}$ (recovering the main result of [14]). The constant $C_{k}$ is explicit (contrary to the constant of [14]) and grows exponentially when $k \rightarrow \infty$. This result has been then improved by Liu [21], where a constant $C_{k}=O\left(k^{2}\right)$ has been obtained. As observed by Funano [13], a positive answer to the open question stated above would yield that under (5.1) the ratios $\lambda^{(k+1)} / \lambda^{(k)}$ are bounded from above by a universal constant.

\section{REFERENCES}

[1] S. Aida and D. Stroock. Moment estimates derived from Poincaré and logarithmic Sobolev inequalities. Math. Res. Lett., 1(1):75-86, 1994.

[2] Luigi Ambrosio and Roberta Ghezzi. Sobolev and bounded variation functions on metric measure spaces. In Geometry, analysis and dynamics on sub-Riemannian manifolds. Vol. II, EMS Ser. Lect. Math., pages 211-273. Eur. Math. Soc., Zürich, 2016.

[3] Kendall Atkinson and Weimin Han. Spherical harmonics and approximations on the unit sphere: an introduction, volume 2044 of Lecture Notes in Mathematics. Springer, Heidelberg, 2012.

[4] D. Bakry and Michel Émery. Diffusions hypercontractives. In Séminaire de probabilités, XIX, 1983/84, volume 1123 of Lecture Notes in Math., pages 177-206. Springer, Berlin, 1985.

[5] Dominique Bakry, Ivan Gentil, and Michel Ledoux. Analysis and geometry of Markov diffusion operators, volume 348 of Grundlehren der Mathematischen Wissenschaften [Fundamental Principles of Mathematical Sciences]. Springer, Cham, 2014.

[6] S. Bobkov and M. Ledoux. Poincaré's inequalities and Talagrand's concentration phenomenon for the exponential distribution. Probab. Theory Related Fields, 107(3):383-400, 1997.

[7] Peter Buser. A note on the isoperimetric constant. Ann. Sci. École Norm. Sup. (4), 15(2):213-230, 1982.

[8] Isaac Chavel. Eigenvalues in Riemannian geometry, volume 115 of Pure and Applied Mathematics. Academic Press, Inc., Orlando, FL, 1984. Including a chapter by Burton Randol, With an appendix by Jozef Dodziuk.

[9] J. Cheeger. Differentiability of Lipschitz functions on metric measure spaces. Geom. Funct. Anal., 9(3):428-517, 1999.

[10] F. R. K. Chung, A. Grigor/yan, and S.-T. Yau. Eigenvalues and diameters for manifolds and graphs. In Tsing Hua lectures on geometry $\mathcal{G}$ analysis (Hsinchu, 1990-1991), pages 79-105. Int. Press, Cambridge, MA, 1997.

[11] F. R. K. Chung, A. Grigor'yan, and S.-T. Yau. Upper bounds for eigenvalues of the discrete and continuous Laplace operators. Adv. Math., 117(2):165-178, 1996.

[12] Joel Friedman and Jean-Pierre Tillich. Laplacian eigenvalues and distances between subsets of a manifold. J. Differential Geom., 56(2):285-299, 2000. 
[13] Kei Funano. Estimates of eigenvalues of the Laplacian by a reduced number of subsets. Israel J. Math., 217(1):413-433, 2017.

[14] Kei Funano and Takashi Shioya. Concentration, Ricci curvature, and eigenvalues of Laplacian. Geom. Funct. Anal., 23(3):888-936, 2013.

[15] Nathael Gozlan, Cyril Roberto, and Paul-Marie Samson. From dimension free concentration to the Poincaré inequality. Calc. Var. Partial Differential Equations, 52(3-4):899-925, 2015.

[16] M. Gromov and V. D. and Milman. A topological application of the isoperimetric inequality. Amer. J. Math., 105(4):843-854, 1983.

[17] Juha Heinonen. Lectures on Lipschitz analysis, volume 100 of Report. University of Jyväskylä Department of Mathematics and Statistics. University of Jyväskylä, Jyväskylä, 2005.

[18] M. Kirszbraun. Uber die zusammenziehende und lipschitzsche transformationen. Fundamenta Mathematicae, 22:77-108, 1934.

[19] Michel Ledoux. The concentration of measure phenomenon, volume 89 of Mathematical Surveys and Monographs. American Mathematical Society, Providence, RI, 2001.

[20] André Lichnerowicz. Géométrie des groupes de transformations. Travaux et Recherches Mathématiques, III. Dunod, Paris, 1958.

[21] S. Liu. An optimal dimension-free upper bound for eigenvalue ratios. ArXiv e-prints, May 2014.

[22] E. J. McShane. Extension of range of functions. Bull. Amer. Math. Soc., 40(12):837-842, 1934.

[23] E. Milman. Spectral Estimates, Contractions and Hypercontractivity. ArXiv e-prints, August 2015.

[24] Emanuel Milman. On the role of convexity in isoperimetry, spectral gap and concentration. Invent. Math., 177(1):1-43, 2009.

[25] Emanuel Milman. Isoperimetric and concentration inequalities: equivalence under curvature lower bound. Duke Math. J., 154(2):207-239, 2010.

[26] Michael Schmuckenschläger. Martingales, Poincaré type inequalities, and deviation inequalities. $J$. Funct. Anal., 155(2):303-323, 1998.

[27] Feng-Yu Wang. Functional inequalities for empty essential spectrum. J. Funct. Anal., 170(1):219 $245,2000$.

[28] Hassler Whitney. Analytic extensions of differentiable functions defined in closed sets. Trans. Amer. Math. Soc., 36(1):63-89, 1934.

Nathael Gozlan

MAP5 (UMR CNRS 8145), UNIVERSité PARIS Descartes.

45 rue des Saints-Pères, 75270 Paris cedex 6, France.

natael.gozlan@parisdescartes. fr

Ronan Herry

MRU, Université DU LuxembourG.

6 avenue de la Fonte, L-4363 Esch-sur-Alzette, Luxembourg.

and

LAMA (UMR CNRS 8050), Université Paris Est Marne la Vallée.

5 bd Descartes, 77454 Marne la Vallée Cedex 2, France.

ronan.herry@uni. lu 